Mitteilungen der Österreichischen Geographischen Gesellschaft, 161. Jg., S. 351-356

(Annals of the Austrian Geographical Society, Vol. 161, pp. 351-356

Wien (Vienna) 2019, https://doi.org/10.1553/moegg161s351

\title{
ZUR NUMISMATISCHEN WÜRDIGUNG DER VON MARTIN WALDSEemülLER GESCHAFFENEN WELTKARTE AUS DEM JAHR 1507
}

\author{
Martin LeHMANN (Freiburg im Breisgau) und Georg SCHIFKO (Wien)*1)
}

mit 2 Abb. im Text

Kaum ein kartographisches Werk hat auch in der breiten Öffentlichkeit so großes Interesse hervorgerufen wie die im lothringischen St. Didel - dem heutigen St. Dié - am „Gymnasium Vosagense“ im Holzdruckverfahren hergestellte Weltkarte des Martin WaLDSEEMÜLLER (Abb. 1). ${ }^{2}$

Diese für ein kartographisches Werk des frühen 16. Jahrhunderts durchaus beachtliche Breitenwirkung war vornehmlich der Tatsache geschuldet, dass auf ihm zu Ehren des Florentiners Amerigo Vespucci erstmals die Bezeichnung „America“ für den neu entdeckten Erdteil jenseits des atlantischen Ozeans Verwendung gefunden hatte. Dieser Umstand verleiht der Weltkarte WALDSEEMÜLLERs aus Sicht der Vereinigten Staaten von Amerika sozusagen identitätsstiftenden Charakter, weshalb sie dort auch als Geburtsurkunde oder Taufschein für die eigene Nation angesehen wird. ${ }^{3)}$

Nachdem dieses kulturhistorisch höchst bedeutsame Dokument im Jahr 1901 vom Jesuitenpater Joseph Fischer in der fürstlichen Bibliothek von Waldburg-Wolfegg (Oberschwaben) in ein Buch eingebunden - und dazu noch in einem hervorragenden Erhaltungszustand - aufgefunden worden

1) Wir danken Herrn Betriebswirt (FH) Falk LieBnitzky für seine Auskünfte bezüglich der Gedenkmünze und der CIT Coin Invest AG für die zur Verfügung gestellten Abbildungen. Ebenso danken wir Dipl.-Ing. Wolfgang MüLLER für seine Unterstützung beim Verfassen des Beitrags.

2) Martin WALDSEEMÜLLER wurde zwischen 1472 und 1475 im kleinen, nur wenige Kilometer von Freiburg im Breisgau entfernt liegenden Weinbauort Wolfenweiler - heute ein Ortsteil der Gemeinde Schallstadt - geboren (LeHMANN 2010, S. 52-56). Der Schöpfer der berühmten Weltkarte absolvierte an der Albert-Ludwigs-Universität Freiburg sein Studium und erwarb sich im weiteren Verlauf seines Lebens auch Kenntnisse im Bereich des Druckerhandwerks. Zu seinen wichtigsten Lehrern an der Universität zählte kein Geringerer als der weit über die Grenzen des Breisgaus hinaus bekannte Kartäuserprior Gregor REISCH, dessen Klosterbibliothek im Dreisamtal zeitweise den Mittelpunkt des geistigen Lebens im südwestdeutschen Raum bildete und als Ort zahlreicher wissenschaftlicher Treffen fungierte. Als Autor der „Margarita Philosophica“, einer vornehmlich nach den „Septem Artes Liberales“ gegliederten Enzyklopädie, war der Berater und spätere Beichtvater Maximilians I. von Habsburg geradezu prädestiniert, seinem Schüler die auf antiken und mittelalterlichen Grundlagen basierenden wissenschaftlichen Voraussetzungen als solide Grundlage für seine kosmographischen und kartographischen Arbeiten zu vermitteln (vgl. HeInZER 2014). Gestorben ist Martin WALdSEEMÜlLER am 16. März 1520 in St. Dié (Ronsin 2006, S. 265, Anm. 51).

3) Eigentlich wird mit dem Eintrag „America“ auf der WALDSEEMÜLLER-Karte Südamerika bezeichnet. Erst Gerhard Mercator hat im Jahr 1538 den Namen auf den ganzen Erdteil ausgeweitet.

* Priv.-Doz. Dr. Martin Lehmann, Albert-Ludwigs-Universität Freiburg, Seminar für Griechische und Lateinische Philologie, Platz der Universität 3, D-79085 Freiburg i. Br.; Dr. Georg SchIFKo, Institut für Kultur- und Sozialanthropologie, Universität Wien, Universitätsstraße 7/4, A-1010 Wien; E-Mails: martin.lehmann3@ gmx.net, georg.schifko@univie.ac.at. 


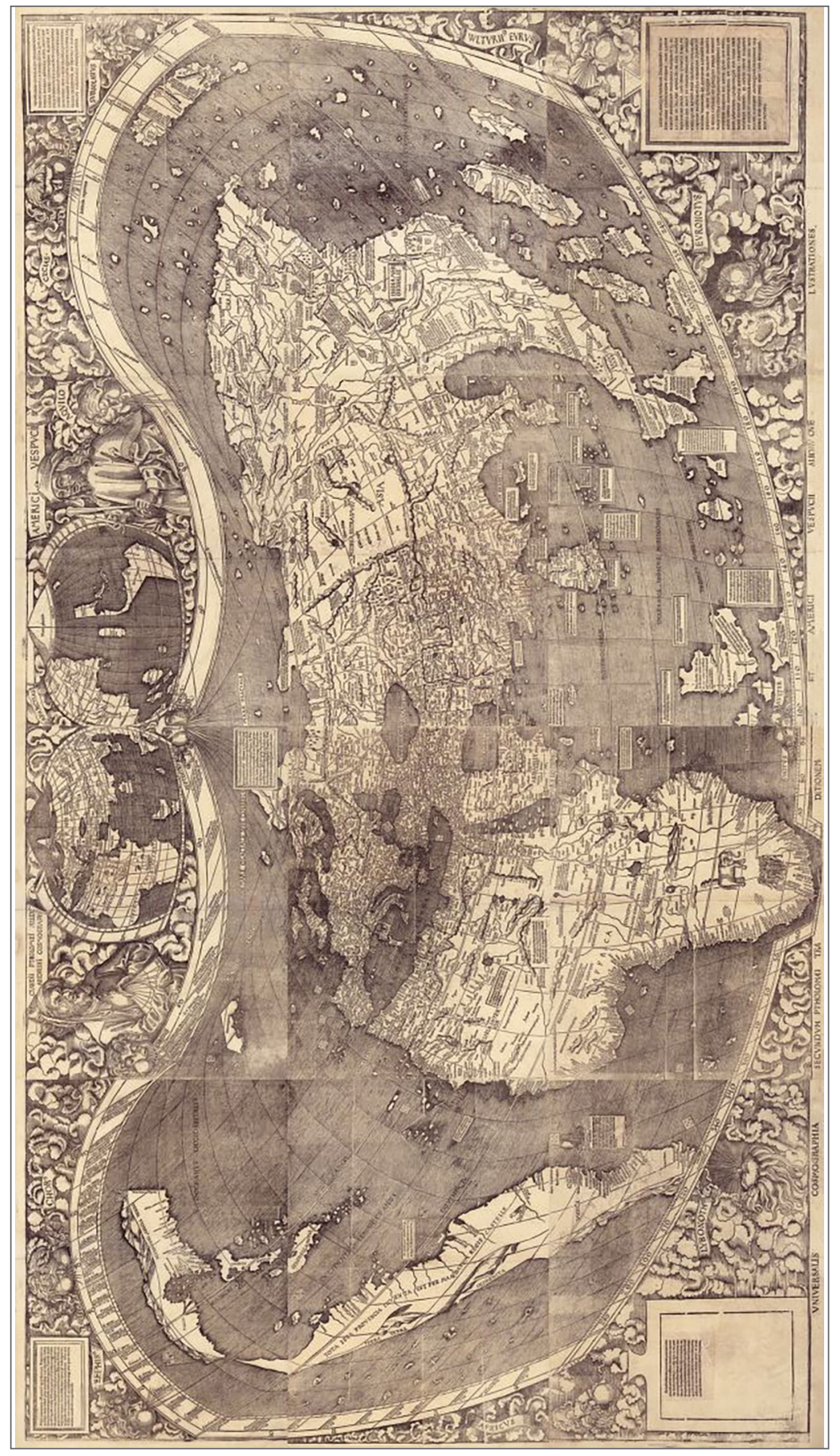

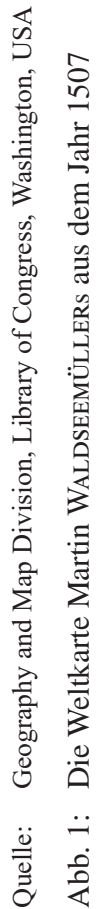


war, ist es der Library of Congress in Washington nach jahrzehntelangen Bemühungen und gegen den Widerstand zahlreicher Fachleute aus dem In- und Ausland im Jahre 2001 schließlich gelungen, diesen unikalen Meilenstein frühneuzeitlicher Kartographie für geschätzte zehn Millionen Dollar käuflich zu erwerben. $\left.{ }^{4}\right)$

Die berühmte, beinahe $3 \mathrm{~m}^{2}$ große Weltkarte war ursprünglich Bestandteil eines am 25. April 1507 in St. Dié veröffentlichten Medienpakets, das außerdem aus einer kleinen, zu einem Globus faltbaren Globussegmentkarte und einer als „Cosmographiae Introductio“ bekannten, von dem aus dem Elsass stammenden Matthias Ringmann verfassten Begleitschrift besteht.5) Im Anhang dieser Einführung in die Kosmographie befinden sich zudem die vier von Amerigo VesPuCCI durchgeführten Entdeckungsfahrten in lateinischer Übersetzung.

Obgleich die aus einem vielfältigen Mosaik kartographischer und literarischer Quellen zusammengefügte Weltkarte WALDSEEMÜLLERs als eine ausschließlich geographisch orientierte Zusammenschau von antikem geographischen Wissen und empirischen Erkenntnissen der frühneuzeitlichen Entdecker auf den Plan tritt, ist die Entstehung dieses kartographischen Meisterwerks dennoch weniger dem humanistischen Bedürfnis nach wissenschaftlichem Erkenntnisfortschritt geschuldet, sondern steht in sehr viel stärkerem Maß mit dem sich zu Beginn des 16. Jahrhunderts zuspitzenden Konflikt zwischen der portugiesischen und der kastilischen Krone um die Vormachtstellung im Gewürzhandel mit Indien im Zusammenhang (vgl. LeHMANN 2016).

Weder entspricht dabei die fälschlicherweise mit VESPUCCI in Verbindung gebrachte separate Amerika-Darstellung den zeitgenössischen geographischen Vorstellungen über die Neue Welt noch gibt die noch immer nach ptolemäischen Vorgaben gestaltete Wiedergabe des Indischen Ozeans und der indischen Gewürzländer den tatsächlichen Wissensstand zu Beginn des 16. Jahrhunderts für diese wirtschaftlich so wichtigen Gebiete wieder. ${ }^{6)}$ Vor diesem Hintergrund stellt die WALDSEEMÜLLER-Karte von 1507 in erster Linie ein politisches Kampfinstrument zur Wahrung der wirtschaftlichen Interessen der Portugiesen ebenso wie derjenigen der Fugger und Maximilians I. von Habsburg im sich zu Beginn des 16. Jahrhunderts anbahnenden Konflikt mit dem kastilischen Königshaus dar.

Im Jahr 2018 erfolgte nun eine numismatische Würdigung dieser historisch bedeutsamen Karte, denn die zu Polynesien gehörenden Cook-Inseln haben eine silberne Gedenkmünze ${ }^{7)}$ ausgegeben (Abb. 2), auf der die Karte gezeigt wird. Besagte Münze ist in einer Auflage von 1500 Stück emittiert worden und jedes Exemplar besteht aus $30 \mathrm{~g}$ extrem dünn gewalztem Silber, das auf der Münzvorderseite geprägt und beiderseits bedruckt ist. Die wie eine mehrfach eingerissene Note wirkende Münze weist die Maße 200 x 150 mm auf. Jede dieser erstaunlicherweise sogar biegsamen Münzen ist zu ihrem Schutz laminiert.

Auf der Münzvorderseite (Avers) ist Königin Elisabeth II. abgebildet, in der Legende wird ein Nominalwert von 5 Dollar angegeben und die Cook-Inseln als Münzherr ausgewiesen. ${ }^{8)}$ Diese Münzseite erinnert insbesondere aufgrund ihrer für moderne Geldscheine typischen geometrischen

4) Aus einem Text auf der „Carta Marina“ aus dem Jahre 1516 - ebenfalls ein kartographisches Meisterstück WALDSEEMÜLLERs - ist die Information zu entnehmen, dass die Weltkarte von 1507 eine Auflage von 1000 Exemplaren hatte.

5) Zum Medienpaket von St. Dié vgl. Lehmann (2010).

6) Amerigo VesPuCCI war der Meinung, dass der neu entdeckte Erdteil in räumlicher Verbindung mit Asien steht (vgl. LeHMANn 2013). Obwohl WALDSEEMÜLLER beispielsweise die auf portugiesischen Quellen basierende CAVERI-Karte (1503) zur Verfügung stand, verschleiert er mit der ptolemäischen Darstellung des Indischen Ozeans die tatsächlichen geographischen Verhältnisse.

7) Die Terminologie solcher Gegenwartsprägungen ist (noch) nicht festgelegt. Ein derartiges Objekt wird bisweilen als „Banknote in Silber“ angesprochen. Im Englischen bezeichnet man es z. B. auch als ,,silver coin note“.

8) Da die Münze aus 30 g Silber besteht, liegt der Nominalwert unter dem Materialwert. 


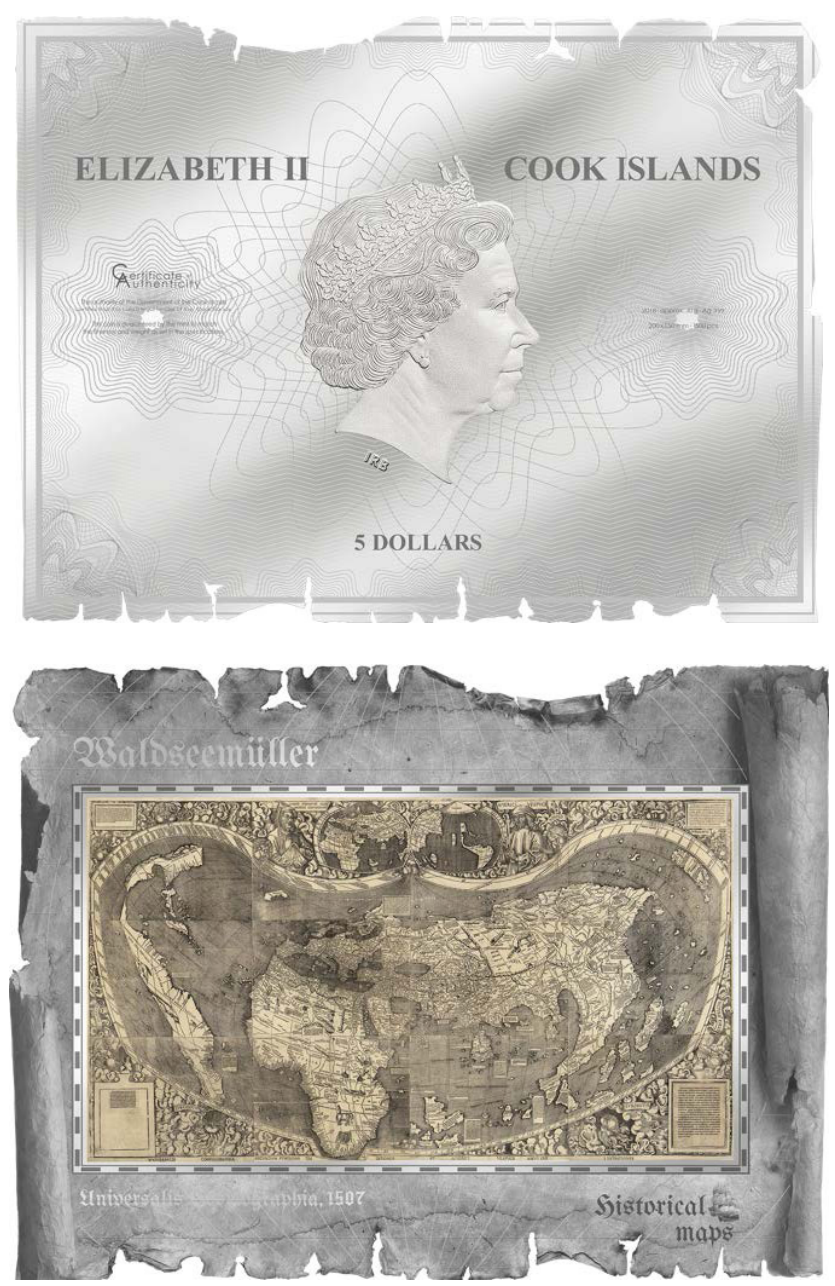

Quelle: CIT Coin Invest AG, Liechtenstein

Abb. 2: Vorderseite (oben) und Rückseite (unten) der Gedenkmünze mit der von WALDSEEMÜLLER 1507 geschaffenen Weltkarte

Muster an Banknoten. Der Aufdruck auf der Rückseite (Revers) der Münze zeigt die in einem Pergament integrierte Karte. Das an den Rändern eingerissene und auf der rechten Seite eingerollte Pergament erweckt, wie bei einem Trompe-l'œil, die Illusion eines dreidimensionalen Objekts. Unter der Karte steht „Universalis Cosmographia 1507“ geschrieben. Rechts unten ist „Historical maps“, das Motto der Münzserie, erkennbar, deren erster und zur Zeit allerdings auch einziger Vertreter die vorliegende Münze ist.

Eine ionische Silbermünze aus dem 4. Jahrhundert v. Chr. weist die vielleicht weltweit älteste Kartendarstellung eines Geldstücks auf, denn in ihrem Gepräge soll in Form eines kartographischen 
Reliefs die Umgebung von Ephesos dargestellt sein (Johnston 1967, S. 86). Gegenwärtig findet man auf mehreren Münzen Karten als Motiv. So geben auch bei uns die 1- und 2-Euro-Münzen auf der Wertseite eine Europakarte wieder, ${ }^{9)}$ während die 1-, 2- und 5-Cent-Münzen auf ihrer Wertseite einen Globus abbilden, auf dem man Afrika, Teile Asiens und - allerdings nur sehr leidlich - auch Europa erkennen kann.

Münzen, die speziell historische Karten zeigen, sind hingegen ziemlich selten. So gibt zum Beispiel eine kugelförmige (!), 2015 in Niue (ebenfalls in Ozeanien) emittierte Münze eine Karte wieder, die 1638 vom Holländer Willem Janszoon BLAEU geschaffen wurde. Dieser unorthodoxen Gedenkmünze sind sogar auch Kristalle der Firma Swarovsky an jenen Stellen auf der Karte (bzw. auf dem Globus) appliziert worden, wo sich die sieben, 2007 per Abstimmung gewählten, „neuen Weltwunder" befinden. ${ }^{10)}$

Nun hat sich 2018 zur überschaubar kleinen Gruppe von historische Karten zeigenden Münzen auch jene der Cook-Inseln hinzugesellt. Ein wenig verwunderlich ist hierbei jedoch der Umstand, dass die Gedenkmünze ausgerechnet von einem pazifischen Inselstaat und nicht von Deutschland oder einem Staat des amerikanischen Doppelkontinents emittiert wurde. Die Cook-Inseln waren den Europäern zu Lebzeiten WaLDSEemüLlERs noch nicht einmal bekannt und man wird sie daher vergebens auf seiner Karte suchen.

Eine Anfrage beim Münzverleger „CIT Coin Invest AG“ (Liechtenstein), ergab, dass die Initiative zur Produktion dieser Silbermünzen von besagter Firma und nicht von den Cook-Inseln ausgegangen ist. Letztere waren jedoch bereit, sie unter ihrer Flagge auszugeben. ${ }^{11)}$ Auch das Emissionsjahr 2018 lässt sich keineswegs mit einem Jubiläum Martin WALDSEEMÜLLERs oder seiner Karte in Verbindung bringen. Es wäre eigentlich viel logischer gewesen, wenn zum Beispiel Deutschland im Jahr 2007, zum Anlass der 500jährigen Existenz der Karte, solch eine Gedenkmünze herausgebracht hätte. Ungeachtet dieses Umstands liegt jetzt parallel zur literarischen Rezeption von WaldSEEmüLleRs Person und der Karte, wie sie beispielsweise 1944 durch den österreichischen Schriftsteller Stefan ZwEIG erfolgt ist, nun auch eine numismatische Würdigung vor, die nicht nur passionierte Münzliebhaber, sondern auch einen kartographisch interessierten Personenkreis ansprechen dürfte.

\section{Literaturverzeichnis}

Heinzer F. (2014): Gregor Reisch und seine Margarita Philosophica. In: KrIEg H., LöBbecke F., Ungerer-Heuck K. (Hrsg.): Die Kartause St. Johannisberg in Freiburg im Breisgau. Freiburg i. Br.: Verlag Stadtarchiv Freiburg.

9) Seit 2008 ist z. B. auch Norwegen auf den Münzen zu finden, obwohl dieser Staat nicht zur EU gehört. Es ist daher auch kaum anzunehmen, dass Großbritannien selbst nach einem Brexit aus dem Münzbild verbannt wird. Dafür hat man im Gepräge die geographische Lage Zyperns verändert, da dieses Land in Wirklichkeit weiter östlich liegt, als es auf den Münzen gezeigt wird. Im Vorfeld der Münzemissionen wurde heftig über die Karten diskutiert, da sich z. B. Griechenland hinsichtlich des Küstenverlaufs nicht richtig dargestellt gefühlt hat. Ebenso wurde von manchen Ländern auch eine Wiedergabe ihrer größten Inseln eingefordert, die dann auch erfolgt ist (SCHÖN 2018, S. 15).

${ }^{10)}$ Bei den ,neuen Weltwundern“ handelt sich um Chichén Itzá (Mexiko), die Chinesische Mauer (Volksrepublik China), Cristo Redentor (Brasilien), das Kolosseum (Italien), Machu Picchu (Peru), die Felsenstadt Petra (Jordanien) und den Taj Mahal (Indien). Die Münze weist einen Nominalwert von 7 Dollar auf und wurde in einer Auflage von 1007 Stück ausgegeben (RosAnOwski 2017, S. 312, LR\#8289).

11) Die Cook-Inseln gehören innerhalb Ozeaniens zu den drei fleißigsten Emittenten von Gedenkmünzen (RosANOwSKi 2017, S. 249). Die beiden anderen Staaten sind Fiji und Palau (SchIFKo 2017, S. 157; 2018, S. 121). 
Johnston A. E. M. (1967): The Earliest Preserved Greek Map. A New Ionian Coin Type. In: Journal of Hellenic Studies, 87, S. 86-94.

Lehmann M. (2010): Die Cosmographiae Introductio Matthias Ringmanns und die Weltkarte Martin Waldseemüllers aus dem Jahre 1507. München: Martin Meidenbauer Verlag.

Lehmann M. (2013): Amerigo Vespucci and his Alleged Awareness of America as a Separate Landmass. In: Imago Mundi, 65 (1), S. 15-24.

Lehmann M. (2016): The Depiction of America on Martin Waldseemüller’s World Map from 1507 - Humanistic Geography in the Service of Political Propaganda. In: Cogent - Arts \& Humanities, 3 (1), pp. 1-15. - https://doi.org/10.1080/23311983.2016.1152785.

Ronsin A. ( ${ }^{32006): ~ L e ~ n o m ~ d e ~ l ' A m e r i q u e, ~ L ' i n v e n t i o n ~ d e s ~ c h a n o i n e s ~ e t ~ s a v a n t s ~ d e ~ S a i n t-D i e ́ ~[D e r ~}$ Name Amerika. Die Erfindung der Kanoniker und Gelehrten von Saint-Dié]. Straßburg [Strasbourg]: La Nuée bleue.

Rosanowski L. (2017): Grand Cataloge of Australian and Oceanian Coins 2000-2017. Danzig [Gdansk]: Mint of Gdansk.

SCHIFко G. (2017): Zur ethnographisch unstimmigen Darstellung eines Inka-Prinzen vor einer mesoamerikanischen Pyramide, die auf einer Gedenkmünze der Cook-Inseln gezeigt wird. In: Mitteilungen der Österreichischen Numismatischen Gesellschaft, 57 (2), S. 155-158.

Schiғко G. (2018): Primärgeld in der Populärkultur - Eine ethno-numismatische Betrachtung zu einer fiktiven Vorgeldform in der Donald Duck-Geschichte „Tralla La“ (1954). In: Mitteilungen der Österreichischen Numismatischen Gesellschaft, 58 (2), S. 115-123.

SснӧN G. (2018): Euro-Münzkatalog. Die Münzen der Europäischen Währungsunion 1999-2018. Regenstauf: Verlag Battenberg Gietl.

ZwEIG S. (1944): Amerigo, die Geschichte eines historischen Irrtums. Stockholm: Bermann-Fischer Verlag. 